\section{AB0196 MESENCHYMAL STEM CELLS TUNE THE DIFFERENTIATION OF MYELOID-DERIVED SUPPRESSOR CELLS IN SJÖGREN'S SYNDROME THROUGH INHIBITING IL-12}

Genhong Yao, Jingjing Qi, Zhuoya Zhang, Saisai Huang, Lingyun Sun. The Affiliated Drum Tower Hospital of Nanjing University Medical School, Department of Rheumatology and Immunology, Nanjing, China

Background: The preliminary animal and human studies, including ours, showed that mesenchymal stem cell (MSC) transplantation could reestablish salivary function and reduce lymphocytic infiltration in salivary glands in Sjögren's syndrome (SS). However, the mechanisms of the encouraging results of MSCs on the SS remain to be elucidated. Myeloid-derived suppressor cells (MDSCs) represent an important class of immunoregulatory cells. MDSCs have multiple phenotypes which inhibit $T$ cell responses by multiple mechanisms, and the environment dictates the development of suppressive properties and activation. Previous studies have demonstrated that MDSCs played important roles in systemic lupus erythematosus and rheumatic arthritis. However, the involvement of MDSCs in the immunopathology of SS is largely unknown

Objectives: This study aims to investigate effects of MDSCs in SS progression and the potential association of MDSCs with the therapeutic effects of MSCs in the patients with SS and mice with SS-like symptoms.

Methods: The frequencies of MDSCs in the blood and bone marrow were detected by flow cytometry analysis in SS mice (NOD mice) and patients with SS. The NOD mice were adoptively transferred with MDSCs or treated with anti-Gr1 antibody to eliminate MDSCs. The salivary flow rate was measured and inflammatory infiltration in salivary glands was assessed. MSCs were infused into NOD mice to investigate the effects of MSCs on SS-like syndrome and changes of MDSCs. The percentages of MDSCs in SS patients before and after MSC transplantation were determined.

Results: The percentage of MDSCs increased significantly with the development of SS-like syndrome in NOD mice. The SS-like syndrome exacerbated after transfer of MDSCs, whereas deletion of MDSCs alleviated SS-like syndrome in NOD mice. MSC transplantation enhanced salivary flow rates and decreased lymphocyte infiltration in salivary glands in NOD mice. The percentages of MDSCs were down-regulated after MSC transplantation. MSC reduced IL-12 production in dendritic cells in vitro and in vivo. The IL-12 promoted generation of MDSCs in vitro. The numbers of MDSCs were positively correlated with disease activities of SS patients. The percentages of MDSCs were reduced after MSC transplantation in patients with SS.

Conclusion: 1. MDSC expansion positively correlated with inflammation during the progression of SS. 2. MSC transplantation had beneficial effects in SS mice. 3. IL-12 production by dendritic cells was reduced by MSCs. 4 . The MDSCs expansion was induced by inflammatory IL-12 and reversed by MSC transplantation in SS. Thus, we have revealed a previously unrecognized function of MSC-mediated reduction of MDSCs in suppressing SSlike syndrome through inhibition of IL-12 production by DCs, which provided novel therapeutic strategies to treat patients with SS.

Acknowledgement: This work was supported by the National Natural Science Foundation of China (NSFC) (grant no. 81571583 and 81770061 to GY).

Disclosure of Interests: None declared

DOI: 10.1136/annrheumdis-2019-eular.359

\section{AB0197 \\ VITAMIN D PROTECTS PODOCYTES FROM AUTOANTIBODIES INDUCED INJURY IN LUPUS NEPHRITIS BY REDUCING ABERRANT AUTOPHAGY}

Qi Yu, Zhaohui Zheng, Zhangsuo Liu. The First Affiliated Hospital Of Zhengzhou University, Zhengzhou, China

Background: Recent data suggest that vitamin $D$ insufficiency may play a role in the progression of SLE and the nephropathy such as chronic kidney disease[1]. We previously show that severe vitamin $D$ deficiency increases the risk for moderate to severe disease activity[2]. Moreover, vitamin $D$ has been demonstrated a protective effect on podocyte injury via diverse mechanisms in proteinuric glomerular disease[3-7]. However, there is a paucity of data to demonstrated the mechanisms through which the autophagy functions on the protective role vitamin $D$ plays on podocyte in the pathogenesis of lupus nephritis

Objectives: The aim of this study was to investigate whether vitamin D play a protective role in podocyte injury induced by autoantibodies purified from lupus nephritis (LN) patients serum via autophagic way.
Methods: Autophagic activities of LN patients renal tissues were evaluated under transmission electronic microscope. IgG from patients with LN were purified to induce human podocyte injury and the role of vitamin $D$ in injury was observed. Podocytes were observed under TEM and autophagic activity was evaluated by western blot analysis and qRT-PCR, mRFP-GFP-LC3B adenovirus were infected into HPC in vitro.

Results: Significantly higher autophagic levels were observed in LN patients $(p<0.05)$ and apparently greater autophagic levels in podocytes were shown $(p<0.05)$. Among different classifications of $L N$, Class $V(n=5)$, $I I I+V(n=5)$ and IV+V $(n=5)$ gained higher autophagic levels than Class III $(n=5)$ and $I V(n=5)$. Induced autophagy, which was evident by increased LC3B-II and Beclin 1 level, caused consumption of p62, more autophagosomes observed under TEM, and more LC3B dots observed under confocal microscope in IgG group, along with decreased Nephrin expression, which suggests podocyte injury. Reduction of autophagy as well as alleviated podocyte injury was observed in the $\lg G+V D$ group.

\section{CONCLUSION:}

This study demonstrates that vitamin $\mathrm{D}$ plays a protective role in podocytes injury induced by autoantibodies from LN patients and that appears to be a novel therapy target in $\mathrm{LN}$.

REFERENCES:

[1] Mok CC, Bro ET, Ho LY, Singh RJ, Jannetto PJ: Serum 25-hydroxyvitamin D3 levels and flares of systemic lupus erythematosus: a longitudinal cohort analysis. Clinical rheumatology 2018.

[2] Gao CC, Liu SY, Wu ZZ, Li TF, Gao GM, Liu ZS, Zheng ZH: Severe vita$\min D$ deficiency increases the risk for moderate to severe disease activity in Chinese patients with SLE. Lupus 2016, 25(11):1224-1229.

[3] Vitamin D down-regulates TRPC6 expression in podocyte injury and proteinuric glomerular disease. The American journal of pathology 2013 182(4):1196-1204.

[4] Zhang XL, Guo YF, Song ZX, Zhou M: Vitamin D prevents podocyte injury via regulation of macrophage M1/M2 phenotype in diabetic nephropathy rats. Endocrinology 2014, 155(12):4939-4950.

[5] Guo J, Lu C, Zhang F, Yu H, Zhou M, He M, Wang C, Zhao Z, Liu Z: VDR Activation Reduces Proteinuria and High-Glucose-Induced Injury of Kidneys and Podocytes by Regulating Wnt Signaling Pathway. Cellular physiology and biochemistry : international journal of experimental celIular physiology, biochemistry, and pharmacology 2017, 43(1):39-51.

[6] Chandel N, Ayasolla K, Wen H, Lan X, Haque S, Saleem MA, Malhotra A, Singhal PC: Vitamin $D$ receptor deficit induces activation of renin angiotensin system via SIRT1 modulation in podocytes. Experimental and molecular pathology 2017, 102(1):97-105.

[7] Garsen M, Sonneveld R, Rops AL, Huntink S, van Kuppevelt TH, Rabelink TJ, Hoenderop JG, Berden JH, Nijenhuis T, van der Vlag J: Vitamin D attenuates proteinuria by inhibition of heparanase expression in the podocyte. The Journal of pathology 2015, 237(4):472-481.

Disclosure of Interests: None declared

DOI: 10.1136/annrheumdis-2019-eular.1556

\section{AB0198 THE ROLE OF LOW EXPRESSION OF MIR-127-3P IN THE PATHOGENESIS OF LUPUS NEPHRITIS}

Yin Zhihua, Qu Bo, Ye Zhizhong. Shenzhen Futian Hospital for Rheumatic Diseases, Shenzhen, China

Background: Lupus nephritis (LN) is one of the most severe organ lesions in systemic lupus erythematosus (SLE). Overactivation of the type I interferon (IFN-I) signaling pathway is associated with the pathogenesis of LN. Overexpression of the IFN stimulated genes (ISGs) was found in the kidneys of $L N$ patients, and the absence of the IFN receptor reduced nephritis in lupus prone mice. Abnormal expression of microRNA in renal tissue is involved in the pathogenesis of LN.

Objectives: We aimed to investigate the role of renal microRNA related to $L N$ in the over-activation of the IFN signaling pathway of LN kidney. Methods: The expression of microRNA was determined by Taqman method. Interferon stimulation response element (ISRE) -luciferase reporter gene assay and western blot assay were used to study the function of candidate microRNA in IFN signal transduction pathway. mRNA expression was measured by SYBR green method. Gene expression profile was detected by gene expression microarray. Antagomir and Agomir (chemically modified microRNA mimics and antagomir inhibitors) of candidate microRNAs were used for functional gain and functional loss experiments. 
Results: Among differentially expressed renal microRNAs in LN, miR-127$3 p$ was reduced in renal tissues of patients with $L N$. The miR-127-3p suppressed the fluorescase gene expression of ISRE induced by ISRE, and the phosphorylation of STAT1 and STAT2. By microarray analysis, we found that most ISG was inhibited by miR127-3p in IFN-stimulated Hela cells. The functional deletion of miR-127-3p enhanced the IFN response in human primary mesangial cells, which was manifested by enhanced ISRE mediated reporter gene expression, enhanced STAT2 phosphorylation and increased ISG expression. In addition, we found that JAK1, the upstream tyrosine kinase of STAT1 and STAT2, was a new target molecule for miR-127-3p.

Conclusion: Our study shows that miR-127-3p can inhibit IFN signal transduction by targeting JAK1. The decreased expression of miR-127-3p in the kidney is associated with an overactive IFN response in the renal tissue of patients with LN. Subsequent mouse model studies indicate the therapeutic potential of $\mathrm{miR}-127-3 p$ in treating lupus associated organ damage.

\section{REFERENCES:}

[1] Han X, Wang $Y$, Zhang $X$, et al. MicroRNA-130b Ameliorates Murine Lupus Nephritis Through Targeting the Type I InterferonPathway on Renal Mesangial Cells. Arthritis Rheumatol. 2016 Sep;68(9):2232-43.

[2] Wu L, Qin Y, Xia S, et al. Identification of Cyclin-Dependent Kinase 1 as a Novel Regulator of Type I Interferon Signaling in Systemic Lupus Erythematosus. Arthritis Rheumatol. 2016 May;68(5):1222-32.

Disclosure of Interests: None declared

DOI: 10.1136/annrheumdis-2019-eular.5732

\section{AB0198B BENEFIT OF CRITHIDIA TESTS IN DIAGNOSING CONNECTIVE TISSUE DISEASES}

Abdul Baseer Awan ${ }^{1}$, Shireen Shaffu ${ }^{2}$, Arthur Price ${ }^{3}$, Panagoula Gkargkoula ${ }^{2}$ ${ }^{1}$ University Hospitals of Leicester, Rheumatology, Leicester, United Kingdom;

${ }^{2}$ University Hospitals of Leicester HPB Unit, Leicester, United Kingdom; ${ }^{3}$ University Hospitals of Leicester HPB Unit, Immunology, Leicester, United Kingdom

Background: The diagnosis of systemic lupus erythematosis(SLE) relies on autoantibody testing, including double stranded DNA (dsDNA), the testing of which has evolved over time from the FARR assay through to ELISA/ELiA. dsDNA assays can pick up non-specific single stranded DNA as false positives and can give occasional false negative results. To evaluate the implications of this we analysed our hospital's use of crithidia testing as a confirmatory assay of dsDNA results.

Objectives: Investigate the relation between dsDNA Abs measured by Crithidia test and ELiA. Define the clinical role for Crithidia testing.

Methods: All crithidia tests for an 8 months from January 2017 to August 2017 were reviewed and results of ANA, ENA, dsDNA and complement collected. Data were collected regarding referral to rheumatology and where possible rheumatology clinic letters were reviewed regarding final diagnosis.

Results: One hundred and fourcrithidia tests were undertaken of which 91 were negative and 13 positive.Sixteen of the 104 patients were ANA negative, 18 ANA $1: 100,70$ ANA $>/=1: 400$, and 14 patients had a positive ENA. Positive crithidias had a dsDNA range from 2 to 333 and negative crithidia from $<1$ to 131 however positive crithidias were more likely to have higher dsDNA and ANA titres. Of the 65 patients seen by rheumatology 4 did not have available notes for analysis. Ten of the $13 \mathrm{cri}-$ thidia positive patients were seen by rheumatology, 6 diagnosed as SLE, 1 as TNF induced lupus, 1 MCTD, 1 RA and 1 had no clinical evidence of autoimmune disease. 88 patients were dsDNA positive (cut off of $>1$ $=9$ ) of whom 11 were also crithidia positive. Of the 13 positive crithidia results, 2 had a negative dsDNA level, one of whom was ANA $1 ; 400$ Ro positive and diagnosed with RA, the other was diagnosed as TNF induced lupus. Of the crithidia negative patients the median dsDNA level was 20 , a range of diagnoses were made including 5 patients with SLE who had a positive Ro or La, 3 patients with SLE who were ENA negative, 4 UCTD, 2 MCTD, 2 with GCA/PMR, 3 with RA and 5 with inflammatory arthropathy. Twenty patients were documented as having no evidence of connective tissue disorders (CTDs), others remain under assessment.

Conclusion: We have shown a significant number of patients using our ELiA assay are dsDNA positive and crithidia negative. In patients with crithidia positivity autoimmune disease is more likely to be diagnosed. Crithidia testing appears to influence whether or not a patient will be referred to rheumatology, with negative crithidias less likely to be referred. This highlights some of the limitations of dsDNA ELISA testing however there is a clear role for this assay as depicted above in preventing over diagnosis of CTDs and unnecessary commitment to immunosuppression. Disclosure of Interests: None declared

DOI: 10.1136/annrheumdis-2019-eular.3953

\section{Systemic sclerosis, myositis and related syndromes - etiology, pathogenesis and animal models}

\begin{tabular}{|l|l}
\hline AB0199 & ASSESSING DISABILITY AND QUALITY OF LIFE IN \\
ALGERIAN SYSTEMIC SCLEROSIS PATIENTS: \\
CONSTRUCT VALIDITIES OF THE ARAB HAND \\
FUNCTION INDEX AND HEALTH ASSESSMENT \\
QUESTIONNAIRE (HAQ)
\end{tabular}

Nouria Benmostefa ${ }^{1,2,3}$, Samy Slimani ${ }^{4}$, Samir Raoubhia ${ }^{4}$, Daoud Roula $^{5}$, Luc Mouthon ${ }^{6}$, Rechid Malek ${ }^{1}{ }^{1}$ Ferhat-Abbas Sétif 1 University, internal medicine, sétif, Algeria; ${ }^{2}$ Sétif Hospital University, internal medicine, Sétif, Algeria; ${ }^{3}$ Cochin Hospital, internal medicine, Paris, France; ${ }^{4}$ Mostefa-Ben Boulaïd Batna University, Rhumathology, Batna, Algeria; ${ }^{5}$ Salah-Boubnider Constantine 3 University, internal medicine, Constantine, Algeria; ${ }^{6}$ National Referral Center for Rare Systemic and Autoimmune Diseases, Hôpital Cochin, Assistance Publique-Hôpitaux de Paris, Université Paris Descartes, Paris, France, Internal medicine, Paris, France

Objectives: To assess the construct validity of the Arab Hand Function Index (AHFI), an Arabic and adapted version of the Cochin hand Functional scale (CHFS) and an Arabic version of the Health Assessment Questionnaire (HAQ) in SSc.

Methods: We evaluated 100 patients with SSc followed between 2015 and 2017. All patients were white and fulfilled the American College of Rheumatology/European League Against Rheumatism criteria and/or the Leroy and Medsger (2) criteria for SSc. Mean \pm SD age at the time of evaluation was $47.66 \pm 12.28$ years. The mean $\pm S D$ disease duration was $6.54 \pm 6.23$ years. Forty-one $(41 \%)$ patients had diffuse cutaneous SSc and $59(59 \%)$ had limited cutaneous SSc. Global hand and wrist mobility were evaluated using the hand functional index (HFI) and Kapandji index. Global disability and hand disability were assessed by an Arabic and adapted version of the $\mathrm{HAQ}$ and by the Arab hand functional index, an Arabic and adapted version of the CHFS, respectively. Anxiety and depression significant symptoms were assessed with the Arabic version of Hospital Anxiety and Depression Scale (HADS) anxiety and HADS depression. Construct validity was assessed by convergent and divergent validity (Spearman's rank correlation coefficient) and factor analysis.

Results: The AHFI had correct convergent validity with global disability assessed by the HAQ (0.61) and the HFI (0.55), moderate correlation with the Kapandji Index (-0.48: inverse correlation), depression (HADd) $(0.45)$ and anxiety (HADa) (0.42) and no correlation with the disease duration (0.29) and the age (0.19) (Table 1). Factor analysis (Table 2) extracted 2 factors that accounted for $64.45 \%$ of the total variance. The HAQ has a good correlation with hand disability (AHF: 0.61), anxiety (0.57) and the HFI (0.51), a moderate correlation with depression $(0.47)$ and Kapandji index $(-0.46)$ and a little correlation with the age $(0.28)$ and the disease duration $(0.10)$ (Table 1).The factor analysis of the $H A Q$, extracted 1 factor that accounted for $50.64 \%$ of the total variance (Table 2).

Conclusion: In patients with SSc, the AHFI and the Arabic HAQ have good construct validity. The total score of the AHFI explained $61 \%$ of the variance of the $\mathrm{HAQ}$.

Acknowledgement: All the patients who participated in this study

Disclosure of Interests: Nouria Benmostefa: None declared, Samy Slimani: None declared, Samir Raoubhia: None declared, Daoud Roula Speakers bureau: Yes, Luc Mouthon: None declared, Rechid Malek: None declared DOI: 10.1136/annrheumdis-2019-eular.7001

Table 1. Convergent and divergent validities of the AHFI and the HAQ for patients with SSc (correlation with other variables)* $^{*}$

\begin{tabular}{lcc}
\hline Scales & Spearman's correlation Coefficient & P Value \\
\hline AHFI & & \\
Convergent validity & & \\
HAQ & 0.61 & $<0.001$ \\
HFI & 0.55 & $<0.001$ \\
Kapandji & -0.48 & $<0.001$ \\
Divergent validity & & \\
Depression (HADd) & 0.45 & $<0.001$ \\
Anxiety (HADa) & 0.42 & $<0.001$ \\
Disease duration & 0.29 & 0.003 \\
Age & 0.19 & 0.058
\end{tabular}

